Gabriella Bené Barbosa ${ }^{1}$

Anna Karlla Sampaio Correia ${ }^{2}$

Luciana de Matos Mota Oliveira ${ }^{3}$

Viviane do Carmo Santos ${ }^{3}$

Sandra Márcia da Silva Ferreira ${ }^{4}$

Davi Félix Martins Júnior ${ }^{5}$

Carlito Lopes Nascimento Sobrinho ${ }^{6}$

\section{Trabalho e saúde mental dos profissionais da Estra- tégia Saúde da Família em um município do Estado da Bahia, Brasil}

\author{
Mental health and work of Family Health Strategy professionals \\ from a municipal district of Bahia State, Brazil
}

${ }^{1}$ Mestranda do Programa de Pós-Graduação em Saúde Coletiva, Departamento de Saúde, Universidade Estadual de Feira de Santana (UEFS). Professora da Área de Odontologia Social do Curso de Odontologia da UEFS.

Feira de Santana, BA, Brasil.

${ }^{2}$ Estudante do Curso de Medicina da Universidade Estadual de Feira de Santana (UEFS). Bolsista Probic/CNPq/UEFS. Feira de Santana, BA, Brasil.

${ }^{3}$ Estudantes do Curso de Medicina da Universidade Estadual de Feira de Santana (UEFS). Bolsistas Pibic/CNPq/UEFS. Feira de Santana, BA, Brasil.

${ }^{4}$ Estudante do Curso de Odontologia da Universidade Estadual de Feira de Santana (UEFS). Feira de Santana, BA, Brasil.

${ }^{5}$ Professor Assistente do Departamento de Saúde, Universidade Estadual de Feira de Santana (UEFS). Vice-Coordenador da Sala de Situação e Análise Epidemiológica e Estatística. Feira de Santana, BA, Brasil.

${ }^{6}$ Professor Titular do Departamento de Saúde, Universidade Estadual de Feira de Santana (UEFS) . Professor do Programa de Pós-Graduação em Saúde Coletiva. Coordenador da Sala de Situação e Análise Epidemiológica e Estatística. Feira de Santana, BA, Brasil.

Contato:

Gabriella Bené Barbosa

Universidade Estadual de Feira de Santana (UEFS)

Avenida Transnordestina, $\mathrm{s} / \mathrm{n}$,

Bairro Novo Horizonte, Campus Universitário, Departamento de Saúde, Feira de Santana, Bahia, Brasil.

CEP: 44031-460

E-mail:

gbenebarbosaster@gmail.com

"Este trabalho foi apoiado e financiado pela Fapesb (PPP0078/2010) e pelo CNPq (473870/2009-6).

Recebido: 04/01/2012

Revisado: 22/08/2012

Aprovado: 24/09/2012

\section{Resumo}

As características do trabalho na Estratégia Saúde da Família (ESF) são complexas e ainda há poucos estudos que abordam a temática. Objetivo: descrever características sociodemográficas e do trabalho e a prevalência de Distúrbios Psíquicos Menores (DPM) e de positivos ao teste CAGE (abuso no consumo de álcool) entre médicos, enfermeiros e cirurgiões-dentistas da ESF de Feira de Santana, Bahia. Metodologia: estudo epidemiológico de corte transversal que utilizou questionário padronizado, não identificado, para descrever: o perfil sociodemográfico; as características do trabalho na ESF; a saúde mental e o uso abusivo de bebidas alcoólicas utilizando o Self Reporting Questionnaire (SRQ-20) e o Teste CAGE,. Os dados foram analisados no Statistical Package for Social Scince (SPSS). Resultados: Entre os 152 respondentes, que correspondem a uma taxa de resposta de 86,4\%, a prevalência de DPM foi de 16,0\%. Entre os médicos, foi de 17,4\%; entre os enfermeiros, de 15,5\%; e 15,2\% entre os cirurgiões-dentistas. Quanto ao uso abusivo de bebida alcoólica, os médicos apresentaram prevalência de 5,0\% e os cirurgiões-dentistas de 7,7\%. Os resultados apontaram o predomínio do sexo feminino, de adultos jovens, casados, com vínculo cooperativado, carga horária semanal elevada e mais de um vínculo empregatício. Conclusão: A prevalência de DPM e de positivos ao teste CAGE observada na população estudada foi menor do que a encontrada em outros estudos.

Palavras-chave: distúrbio psíquico menor; SRQ-20; Estratégia Saúde da Família; estudo de prevalência; saúde do trabalhador.

\begin{abstract}
To date, few studies have looked into the complex aspects of work at the Family Health Strategy (FHS). Objective: To describe sociodemographics, work aspects, Minor Psychiatric Disorders (MPD), and CAGE positivity (alcoholism) among physicians, nurses and dentists working with FHS in Feira de Santana (Bahia, Brazil). Methods: Cross-sectional study using standard questionnaires (SRQ20 and CAGE) to collect anonymous information on the sociodemographic profile, work aspects, mental health, and alcohol consumption. Data were analyzed with SPSS. Results: Based on 152 respondees (response rate: $86.4 \%$ ), the overall prevalence of MPD was $16.0 \%$ (physicians $=17.4 \%$, nurses $=15.5 \%$, dentists $=15.2 \%$ ). The prevalence of alcoholism was $5.0 \%$ among physicians and $7.7 \%$ among dentists. The characteristics "female", "young adult", "married", "affiliated to a cooperative", "long work week" and "more than one job" predominated in this population. Conclusion: the prevalence of MPD and CAGE positivity was smaller in our sample than in other similar studies.
\end{abstract}

Keywords: minor psychiatric disorder; SRQ-20; Family Health Strategy; prevalence study; occupational health. 


\section{Introdução}

O trabalho humano possui um duplo caráter: por um lado é fonte de realização, satisfação, prazer, estruturando e conformando o processo de identidade dos sujeitos; por outro, pode também se transformar em elemento patogênico, tornando-se nocivo à saúde (DEJOURS, 1987). Entretanto, ao longo do tempo, o trabalho tem representado fonte de dor, adoecimento e morte devido a diferentes formas de exploração a que o homem tem se submetido.

Segundo Antunes (1995), a ampliação das atividades de trabalho vem interferindo na qualidade de vida, saúde e segurança dos trabalhadores. Kurz (2005) afirmou que os efeitos dessa realidade são potencializados pela intensificação do trabalho, pelo aumento da jornada, pela desregulamentação de direitos trabalhistas e pela precarização do trabalho.

A assistência à saúde, como atividade do setor de serviço, transformou-se progressivamente, no sistema de produção capitalista, em uma mercadoria socialmente valorizada, coisificando o trabalho na saúde. Os trabalhadores passaram a submeter-se às regras deste sistema e desenvolver suas atividades em serviços públicos e privados seguindo regras impostas aos trabalhadores de qualquer empresa capitalista (instabilidade no emprego, ritmo intenso de trabalho, jornadas de trabalho prolongadas), somadas às particularidades do trabalho na saúde, como aliviar a dor e o sofrimento (NASCIMENTO SOBRINHO; NASCIMENTO; CARVALHO, 2005).

O Programa Saúde da Família (PSF) foi criado em 1994 pelo Ministério da Saúde. Atualmente chamado Estratégia Saúde da Família (ESF), é um modelo prioritário para a reorganização da atenção básica à saúde no Brasil, configurando-se como porta de entrada ao Sistema Único de Saúde (SUS). Trata-se de uma prática transformadora, na qual a atenção à saúde está centrada na família, entendida e percebida a partir de seu ambiente físico e social, que tem como diretriz operacional: os princípios de caráter substitutivo de suas práticas; a integralidade e a hierarquização das ações; a territorialização e a definição da clientela; e a adoção do trabalho em equipe multiprofissional (BRASIL, 1997).

O processo de trabalho na ESF envolve a execução de diversas atividades através da divisão de tarefas, tornando-o um processo bastante complexo. A relação de trabalho baseada na interdisciplinaridade e não mais na multidisciplinaridade, associada à não aceitação do refúgio da assistência no positivismo biológico, requer uma abordagem que questione as certezas profissionais e estimule a permanente comunicação horizontal entre os componentes da equipe. Exige-se, assim, um profissional com visão sistêmica e integral do indivíduo, da família e da comunidade, capaz de atuar com criatividade e senso crítico mediante uma prática humanizada e resolutiva, envolvendo ações de promoção, proteção, cura e reabilitação. O profissional deve estar capacitado para planejar, organizar, executar e avaliar ações que correspondam às reais necessidades da comunidade, articulando os setores envolvidos na promoção da saúde e interagindo com a comunidade, mobilizando-a a participar de forma dinâmica nas atividades a serem desenvolvidas com avaliação permanente através de indicadores de saúde da área de abrangência (BRASIL, 2001a, 2001b).

Além das exigências inerentes à atenção integral à saúde e à humanização das práticas, os médicos, enfermeiros e cirurgiões-dentistas que atuam nas ESFs ficam expostos a situações geradas pela pobreza, pelas desigualdades sociais e pelas deficiências dos demais níveis do sistema de saúde, desenvolvendo assim múltiplas habilidades e incorporando complexas tecnologias para superar os desafios de seus cotidianos profissionais.

As mudanças e a precarização dos serviços de saúde, que podem gerar medo, isolamento e submissão, e a falta de reconhecimento profissional, aliada à discussão tardia sobre planos de cargos e salários para os trabalhadores do SUS, ao arrocho salarial e ao aumento da demanda por serviços públicos de saúde, constituem-se em um estímulo à investigação das condições de trabalho dessas categorias profissionais, em especial entre aqueles que atuam na Estratégia Saúde da Família (BODSTEIN, 2002).

Para Goldberg e Huxley (1993), a expressão Distúrbio Psíquico Menor (DPM) foi criada para designar sintomas de insônia, fadiga, irritabilidade, esquecimento, dificuldade de concentração e queixas somáticas que demonstram ruptura do funcionamento normal do indivíduo, mas não configuram categoria nosológica da $10^{\underline{a}}$ Classificação Internacional de Doenças (CID-10), nem dos Manuais de Diagnóstico e Estatística (DSM) da Associação Psiquiátrica Americana, mas constituem problema de saúde pública e apresentam impactos econômicos relevantes em função das demandas geradas aos serviços de saúde e do absenteísmo no trabalho (COUTINHO; ALMEIDA-FILHO; MARI, 1999).

Os DPMs, entre os trabalhadores da saúde, podem estar relacionados a: jornadas prolongadas de trabalho; ritmo acelerado de trabalho; quase inexistência de pausa para descanso ao longo do dia; e intensa responsabilidade sobre as tarefas executadas. Alguns estudos revelaram elevada prevalência de DPM entre os profissionais de saúde. Nascimento Sobrinho et al. (2006) encontraram uma prevalência de $26,0 \%$ de DPM em médicos na cidade de Sal- 
vador, BA. Silva et al. (2011) abordaram a relação trabalho-saúde entre trabalhadores de enfermagem de um hospital federal no município do Rio de Janeiro, identificando, no cotidiano do trabalho desses profissionais, situações que acarretam desgaste físico e mental.

Pouco se conhece sobre as características sociodemográficas, a situação profissional e a prevalência de DPMs entre os trabalhadores que atuam na ESF. Na literatura nacional encontramos apenas um estudo que abordou a saúde do trabalhador que atua na Atenção Básica, incluindo a ESF (BRAGA; CARVALHO; BINDER, 2010). O objetivo deste estudo foi descrever as características sociodemográficas, as características do trabalho e a prevalência de DPMs e de profissionais positivos ao teste CAGE (teste de triagem para detecção de abuso no consumo de bebidas alcóolicas) entre médicos, enfermeiros e cirurgiões-dentistas das Unidades de Saúde da Família (USF) de Feira de Santana, BA.

\section{Metodologia}

Foi realizado um estudo epidemiológico populacional, de corte transversal, desenvolvido em todas as USF da cidade de Feira de Santana, BA, no período de maio a setembro de 2011. Foram estudados todos os médicos, enfermeiros e cirurgiões-dentistas, sendo incluídos os que desenvolviam atividades assistenciais há mais de um mês e excluídos os que se encontravam em atividades administrativas, afastados por licença maternidade ou por doença ou em gozo de férias no início da coleta de dados e os que não consentiram participar do estudo após a leitura do Termo de Consentimento Livre e Esclarecido (TCLE). Foram considerados perda aqueles profissionais que trabalhavam na ESF, mas não foram encontrados e os que inicialmente concordaram em participar do estudo, mas não devolveram o questionário.

O município de Feira de Santana é a segunda maior cidade do Estado, com $1.362 \mathrm{~km}^{2}$, dividido em 88 bairros e 08 distritos, com uma população aproximada de 600.000 habitantes. A implantação da ESF no município foi em novembro de 2000, atualmente possui 77 USF, com 83 Equipes de Saúde da Família, cobrindo 79,6\% da população, e 42 Equipes de Saúde Bucal - ESB - [FEIRA DE SANTANA (Município), 2006].

O instrumento de coleta de dados utilizado foi um questionário padronizado, não identificado, contendo três escalas validadas - Job Content Questionnaire (JCQ), Self Reporting Questionnaire (SRQ-20) e Teste CAGE -, composto de dez blocos buscando conhecer: $1^{\circ}$ ) o perfil sociodemográfico dos entrevistados; $2^{\circ}$ ) as informações gerais sobre o trabalho na ESF; $3^{\circ}$ ) o ambiente de trabalho; $4^{\circ}$ ) os aspectos psicossociais do trabalho, utilizando o JCQ; $5^{\circ}$ ) as atividades domésticas e os hábitos de vida, com questões para detectar o abuso no consumo de bebidas alcoólicas, utilizando o Teste CAGE, e atividades de lazer; $6^{\circ}$ ) a capacidade para o trabalho; $7^{\circ}$ ) os aspectos relacionados à saúde física dos indivíduos para identificar queixas e sintomas de alguns agravos à saúde e doenças diagnosticadas após o ingresso na ESF; 8º) a saúde mental dos trabalhadores, utilizando o SRQ-20 para detecção de DPM; 9º) as informações sobre atos de violência - vitimização; e $10^{\circ}$ ) as questões sobre padrões de sono. Destes dez blocos, foram utilizadas, neste estudo, questões para conhecer o perfil sociodemográfico dos entrevistados, os hábitos de vida, as características da situação profissional e a prevalência de DPM e de profissionais positivos ao teste CAGE.

O SRQ foi desenvolvido por Harding et al. (1980) sob a coordenação da Organização Mundial de Saúde (OMS) e validado para utilização no Brasil por Mari e Willians (1986), com a finalidade de estudar a morbidade psiquiátrica em instituições de saúde. A versão SRQ-20 é a mais utilizada em estudos de base populacional, sendo composta de 20 questões: 04 sobre sintomas físicos e 16 sobre sintomas psicoemocionais. As respostas são do tipo "sim" ou "não", atribuindo-se, respectivamente, valores de "1" e "0". O ponto de corte sugerido pelos autores para a suspeita de DPM é o de 07 respostas positivas. $\mathrm{O}$ trabalhador que apresentou escore $\geq 07$ respostas positivas foi considerado positivo ao SRQ-20.

O teste CAGE é um questionário padronizado, composto de 04 perguntas. Sua denominação é derivada das iniciais das palavras chaves de cada pergunta do original em inglês: 1) Alguma vez sentiu que deveria diminuir ou parar de beber? (Cut down?); 2) As pessoas o aborrecem porque criticam o seu modo de beber? (Annoying by criticism?); 3) Sente-se culpado pela maneira que costuma beber? (Guiltier about drinking?); 4) Costuma beber pela manhã para diminuir o nervosismo e a ressaca? (Eye-opener drink?). O CAGE é utilizado como teste de triagem para detecção de abuso no consumo de bebidas alcoólicas, adotando-se o ponto de corte em duas ou mais respostas positivas para as quatro questões do teste. Apresenta alta sensibilidade, especificidade e valores preditivos tanto na sua versão em inglês, como na versão em português (MASUR; MONTEIRO, 1983). Os indivíduos com o escore $\geq 02$ respostas positivas foram considerados positivos ao teste CAGE. 
Um estudo piloto de corte transversal e base populacional foi realizado no mês de outubro de 2010, em 10 (dez) USF no município de São Gonçalo dos Campos, Bahia, localizado a $20 \mathrm{~km}$ de Feira de Santana, com a finalidade de verificar o tempo aproximado de preenchimento e a clareza do instrumento de coleta de dados. Os sujeitos pesquisados foram 29 profissionais da ESF, sendo 10 de Medicina, 10 de Enfermagem e 09 de Odontologia.

Para o estudo principal, foi solicitada da Secretaria Municipal de Saúde de Feira de Santana a lista dos trabalhadores que atuavam na ESF com a sua distribuição entre as USF. Seguiu-se com a divulgação da pesquisa em todas as USF por meio de cartazes e folhetos para despertar o interesse da população estudada e por meio de reuniões com o objetivo de informar os trabalhadores sobre o projeto e os seus procedimentos.

Os questionários, devidamente numerados, foram entregues aos profissionais nas USF, acompanhados do TCLE, atendendo ao estabelecido pela Resolução 196/96 do Ministério da Saúde. A pesquisadora esclareceu os objetivos do estudo e as instruções gerais o preenchimento, aguardando a sua devolução.

Os trabalhadores que aceitaram participar do estudo preencheram o questionário no seu próprio local de trabalho. Para atender ao trabalhador que, por razões relacionadas à dinâmica do serviço, não podia interromper suas atividades para o preenchimento do instrumento, em comum acordo com sua chefia, visando não causar transtornos, a pesquisadora voltava em outra ocasião para a coleta dos questionários respondidos. Durante a coleta, reuniões semanais foram realizadas com toda a equipe envolvida na pesquisa para entrega e revisão dos questionários coletados.

O projeto foi aprovado pela Divisão da Atenção Básica à Saúde da Secretaria Municipal de Saúde de Feira de Santana e pelo Comitê de Ética em Pesquisa cadastrado no Conep sob o Registro de Protocolo $\mathrm{n}^{\circ}$ 063/2008 (CAAE 0062.059.000-08).

Para confrontar as informações e identificar e corrigir possíveis erros inconsistências de digitação, foi realizada uma dupla digitação dos dados coletados utilizando o programa EpiData for Windows versão 3.1. Para a análise estatística, foi utilizado o programa Statistical Package for Social Science (SPSS) versão 9.0, disponibilizado pela Universidade Estadual de Feira de Santana (UEFS).

Uma análise descritiva univariada dos dados foi realizada a partir do cálculo de frequências absolutas e relativas das variáveis categóricas e da média e do desvio padrão das variáveis numéricas referen- tes às características sociodemográficas, das condições de trabalho e da situação de saúde mental dos trabalhadores.

\section{Resultados}

A ESF no município de Feira de Santana possui 77 unidades e 83 equipes, das quais 05 apresentaram ausência de médicos. Dos 78 médicos inicialmente elegíveis, 48 participaram do estudo, ocorrendo 01 perda, 18 recusas e 11 não atenderam aos critérios de inclusão, sendo contabilizados: 03 de licença médica; 02 de licença maternidade; 04 de férias; 02 recém-contratados (há menos de 01 mês). Dessa forma, obteve-se uma adesão de 71,6\% dos médicos elegíveis (67). Dos 83 enfermeiros inicialmente elegíveis, participaram do estudo 71 (85,5\%), ocorrendo 02 perdas, 03 recusas e 07 não atenderam aos critérios de inclusão, sendo contabilizados: 01 de férias; 02 de licença maternidade e 04 trabalhando há menos de 01 mês na ESF. Dessa forma, obteve-se uma adesão de 91,0\% entre enfermeiros elegíveis (76). Das 77 USF, 42 (21,7\%) possuíam Equipes de Saúde Bucal (ESB), com 33 cirurgiões-dentistas, sendo que 09 atuam em mais de uma USF. Desses 33, obteve-se uma adesão de 100\%.

Dos 194 profissionais das três categorias que atuam na ESF, 18 foram excluídos do estudo por não atenderem aos critérios de inclusão, totalizando 176. Destes, 152 responderam ao questionário, obtendo-se uma taxa de resposta de $86,4 \%$.

Os resultados apontaram que 116 (76,3\%) sujeitos estudados são do sexo feminino. Com relação à idade, a média encontrada foi de $38,4 \pm 13,3$. Na Tabela 1 observa-se, entre os médicos, uma maior frequência do sexo masculino $(54,2 \%)$ e faixa etária maior que 38 anos $(56,2 \%)$. Aproximadamente metade dos médicos que atuam na ESF de Feira de Santana apresenta idade entre 25 e 30 anos e entre 60 e 78 anos (19,1\% e $27,7 \%$ respectivamente). Tais resultados caracterizam uma polarização indicando que a medicina na ESF está sendo exercida por profissionais jovens ou da terceira idade. Nas categorias dos enfermeiros e cirurgiões-dentistas, predominou o sexo feminino, $95,8 \%$ e $78,8 \%$ respectivamente, e a faixa etária menor ou igual a 38 anos, $77,5 \%$ e $57,6 \%$ respectivamente. Quanto à situação conjugal, nas três categorias foi mais frequente a de casados $(51,3 \%)$.

As características da situação profissional das três categorias estão descritas na Tabela 2, que aborda o vínculo atual de trabalho, a carga horária semanal, a renda mensal e se trabalha em outro local. 
Tabela 1 Características sociodemográficas dos trabalhadores que atuam na Estratégia Saúde da Família de Feira de Santana, Bahia, 2011

\begin{tabular}{|c|c|c|c|c|c|c|c|}
\hline \multirow[t]{2}{*}{ Variável } & \multirow[b]{2}{*}{$N^{*}$} & \multicolumn{2}{|c|}{ Médico(a) } & \multicolumn{2}{|c|}{ Enfermeiro(a) } & \multicolumn{2}{|c|}{ Dentista } \\
\hline & & $n$ & $\%$ & $n$ & $\%$ & $n$ & $\%$ \\
\hline Sexo & 152 & & & & & & \\
\hline Masculino & & 26 & 54,2 & 03 & 4,2 & 07 & 21,2 \\
\hline Feminino & & 22 & 45,8 & 68 & 95,8 & 26 & 78,8 \\
\hline Faixa etária & 152 & & & & & & \\
\hline$\leq 38$ anos & & 21 & 43,8 & 55 & 77,5 & 19 & 57,6 \\
\hline$>38$ anos & & 27 & 56,2 & 16 & 22,5 & 14 & 42,4 \\
\hline Situação conjugal & 150 & & & & & & \\
\hline Solteiro(a) & & 08 & 16,7 & 27 & 39,1 & 12 & 36,4 \\
\hline Casado(a) & & 27 & 56,3 & 35 & 50,8 & 15 & 45,5 \\
\hline Outros & & 13 & 27,0 & 07 & 10,1 & 06 & 18,1 \\
\hline \multirow[t]{2}{*}{ Categoria profissional } & 152 & & & & & & \\
\hline & & 48 & 31,6 & 71 & 46,7 & 33 & 21,7 \\
\hline
\end{tabular}

* Respostas válidas, excluídas as respostas ignoradas.

Tabela 2 Características da situação profissional dos trabalhadores que atuam na Estratégia Saúde da Família de Feira de Santana, Bahia, 2011

\begin{tabular}{|c|c|c|c|c|c|c|c|}
\hline \multirow{2}{*}{ Variável } & \multirow[b]{2}{*}{$N^{*}$} & \multicolumn{2}{|c|}{ Médico(a) } & \multicolumn{2}{|c|}{ Enfermeiro(a) } & \multicolumn{2}{|c|}{ Dentista } \\
\hline & & $n$ & $\%$ & $n$ & $\%$ & $n$ & $\%$ \\
\hline Vínculo atual de trabalho & 150 & & & & & & \\
\hline Cooperativado & & 40 & 85,1 & 68 & 97,1 & 32 & 97,0 \\
\hline Outros & & 07 & 14,9 & 02 & 2,9 & 01 & 3,0 \\
\hline Carga horária semanal & 146 & & & & & & \\
\hline$\leq 40$ horas & & 21 & 44,7 & 44 & 66,7 & 16 & 48,5 \\
\hline$>40$ horas & & 26 & 55,3 & 22 & 33,3 & 17 & 51,5 \\
\hline Renda mensal & 131 & & & & & & \\
\hline$\leq 5.000,00$ & & 02 & 5,9 & 63 & 96,9 & 32 & 100,0 \\
\hline$>5.000,00$ & & 32 & 94,1 & 02 & 3,1 & 0,0 & 0,0 \\
\hline Trabalha em outro local & 150 & & & & & & \\
\hline No setor público & & 26 & 56,5 & 11 & 15,5 & 04 & 12,1 \\
\hline No setor privado & & 10 & 21,7 & 11 & 15,5 & 07 & 21,2 \\
\hline Por conta própria & & 02 & 4,4 & 05 & 7,0 & 09 & 27,3 \\
\hline Não trabalha em outro local & & 08 & 17,4 & 44 & 62,0 & 13 & 39,4 \\
\hline
\end{tabular}

* Respostas válidas, excluídas as respostas ignoradas. 
O tipo de vínculo de trabalho mais frequente foi o cooperativado com 93,3\%: entre os médicos $85,1 \%$; enfermeiros $97,1 \%$; e cirurgiões-dentistas $97,0 \%$. Questionados sobre o treinamento para atuar no PSF, 63,9\% informaram que não foram treinados e $88,6 \%$ responderam receber treinamento durante as atividades no PSF. Quanto à carga horária semanal geral (ESF e outros vínculos) de trabalho, 55,3\% dos médicos e 51,5\% cirurgiões-dentistas ultrapassaram 40 horas semanais de trabalho, enquanto que 96,9\% dos enfermeiros apresentam carga horária de trabalho menor ou igual a 40 horas. A maioria dos profissionais pesquisados (98,7\%), nas três categorias, trabalha nos turnos matutino e vespertino e apenas 4,0\% trabalha em regime de plantão em outro local, paralelamente ao trabalho na ESF, sendo destes $70 \%$ médicos e $30 \%$ enfermeiros.

A renda mensal maior que $\mathrm{R} \$ 5.000,00$ (cinco mil reais) foi mais frequente entre os médicos (94,1\%), apresentando uma média de R\$ 9.424,00 (nove mil quatrocentos e vinte e quatro reais). Os enfermeiros $(96,9 \%)$ informaram uma renda menor ou igual a $\mathrm{R} \$ 5.000,00$ (cinco mil reais), com média de $\mathrm{R} \$ 2.732,00$ (dois mil setecentos e trinta e dois reais). Dos cirurgiões-dentistas, 100\% informaram renda menor ou igual a $\mathrm{R} \$ 5.000,00$ (cinco mil reais), com média de R\$ 3.177,00 (três mil cento e setenta e sete reais).

Ao serem questionados se trabalham em outro local, a maioria dos médicos, $82,6 \%$, e dos cirurgiões-dentistas, 60,6\%, respondeu possuir outro vínculo, enquanto que, $62,0 \%$, dos enfermeiros afirmaram não trabalhar em outro local.
As características da situação de saúde das três categorias profissionais estão apresentadas na Tabela 3. O SRQ-20 descreveu a prevalência de DPMs, e o Teste CAGE identificou a prevalência do uso abusivo de bebidas alcoólicas, detectando os profissionais positivos ao teste CAGE.

A prevalência de DPM entre as categorias estudadas foi de 16,0\%, sendo entre os médicos de 17,4\%, entre os enfermeiros de $15,5 \%$ e entre os cirurgiões-dentistas de 15,2\%. Com relação ao uso abusivo de bebida alcoólica, a prevalência geral foi de 1,3\%. Entre os médicos e os cirurgiões-dentistas que informaram fazer uso de bebida alcoólica, a prevalência de positivos ao teste CAGE foi de 5,0\% e 7,7\% respectivamente. Quanto à categoria de enfermagem, não houve profissionais com o teste positivo.

\section{Discussão}

Em Feira de Santana, das 83 ESFs, 05 não apresentam médicos, resultado que aponta uma discordância com o que é preconizado pelo SUS quanto à modalidade mínima de formação das equipes: 01 médico generalista ou da família, 01 enfermeiro, 01 auxiliar de enfermagem e de 04 a 06 agentes comunitários de saúde (BRASIL, 1997).

Nas categorias estudadas, predominou o sexo feminino quanto aos enfermeiros e cirurgiões-dentistas, observando-se semelhança com outros estudos (GOMES, 2011; LONGHI; CRACO, 2011; MORONI; PAZ, 2011), bem como, com os registros de indicadores de gestão do trabalho em saúde, diferindo dos médicos que atuam na ESF (BRASIL, 2007).

Tabela 3 Características da situação de saúde mental e consumo de álcool dos trabalhadores que atuam na Estratégia Saúde da Família de Feira de Santana, Bahia, 2011

\begin{tabular}{|c|c|c|c|c|c|c|c|}
\hline \multirow{2}{*}{ Variável } & \multirow[b]{2}{*}{$N^{*}$} & \multicolumn{2}{|c|}{ Médico(a) } & \multicolumn{2}{|c|}{ Enfermeiro(a) } & \multicolumn{2}{|c|}{ Dentista } \\
\hline & & $n$ & $\%$ & $n$ & $\%$ & $n$ & $\%$ \\
\hline SRQ-20*** & 150 & & & & & & \\
\hline Positivo & & 08 & 17,4 & 11 & 15,5 & 05 & 15,2 \\
\hline Negativo & & 38 & 82,6 & 60 & 84,5 & 28 & 84,8 \\
\hline CAGE䊑*** & 57 & & & & & & \\
\hline Positivo & & 01 & 5,0 & 0 & 0,0 & 01 & 7,7 \\
\hline Negativo & & 19 & 95,0 & 24 & 100,0 & 12 & 92,3 \\
\hline
\end{tabular}


Dos 78 médicos, 48 concordaram em responder os questionários, sendo a maioria do sexo masculino, com idade acima de 38 anos e casados, corroborando os achados de Nascimento Sobrinho et al. (2006) e Barros et al. (2008). Este estudo apresentou uma menor adesão dos médicos quando comparados aos demais profissionais pesquisados. Estes achados coincidem com o de outras pesquisas realizadas com essa categoria profissional, apontando a dificuldade na obtenção de respostas em pesquisas com médicos (BARBOSA et al., 2007; BRUGUERA et al., 2001; MACHADO, 1997; NASCIMENTO SOBRINHO et al., 2006; TUCUNDUVA et al., 2006).

Entre os 83 enfermeiros, 71 aderiram ao estudo e todos os cirurgiões-dentistas responderam ao questionário, sendo a maioria do sexo feminino, com idade menor ou igual a 38 anos e casados, coincidindo com outros estudos (ARAÚJO; GRAÇA; ARAÚJO, 2003; KIRCHOF et al., 2009; MORITA; HADDAD; ARAÚJO, 2010; MORONI; PAZ; 2011; SILVA et al., 2011; SOUSA, 2010).

Para descrever as características da situação profissional, foram coletados dados sobre o atual vínculo de trabalho, a carga horária semanal, a renda mensal e o trabalho em outro local. A maioria dos médicos possui vínculo de trabalho por meio de cooperativa de trabalho, com elevada carga horária semanal, renda mensal superior a das outras categorias estudadas e predomínio do setor público como outro local de trabalho, semelhante aos resultados dos estudos de Nascimento Sobrinho et al. (2006), de Barros et al. (2008) e de Moroni e Paz (2011), configurando-se uma sobrecarga de trabalho para justificar o retorno financeiro, podendo influenciar no seu cotidiano profissional e na qualidade de vida.

Os enfermeiros apresentaram maior frequência de vínculo por meio de cooperativa de trabalho, carga horária semanal menor ou igual a 40 horas, condizente com o que estabelece a ESF, renda mensal inferior a $\mathrm{R} \$ 5.000,00$ (cinco mil reais) e predomínio de único vínculo. Estes resultados são semelhantes aos resultados dos estudos de Araújo, Graça e Araújo (2003), de Kirchof et al. (2009), de Sousa (2010) e de Moroni e Paz (2011). A carga horária observada e o menor número de vínculo de trabalho podem estar relacionados a uma atuação assistencial que exige tempo para interação com o paciente e seu acompanhamento, além de atividades administrativas que são desenvolvidas na USF por essa categoria profissional. Outro aspecto a ser considerado, com base na análise de Chaves e Miranda (2008), é a ESF como oportunidade imediata de "transição" para o trabalho assalariado na esfera pública, em busca de alguma remuneração regular, apesar da precariedade do vínculo de trabalho.
Os cirurgiões-dentistas apresentaram resultados semelhantes aos dos enfermeiros quanto ao vínculo e à renda, diferindo quanto à carga horária semanal e ao trabalho em outro local, neste ponto assemelhando-se a dos médicos, corroborando os achados de Araújo, Graça e Araújo (2003). A renda percebida por essa categoria pode estar associada à redução da remuneração deste profissional, tanto no setor público, como no privado (MORITA; HADDAD; ARAÚJO, 2010). Nesta categoria profissional, a inserção na ESF pode representar oportunidade única e imediata de inserção no mercado de trabalho assalariado na esfera pública, da garantindo remuneração regular, apesar da precarização do vínculo de trabalho, da baixa remuneração e do constrangimento pela dificuldade de permanência na esfera privada (CHAVES; MIRANDA, 2008).

Segundo Nascimento Sobrinho et al. (2004), as cooperativas de trabalho são definidas como sociedades constituídas para prestar serviços a seus associados, sem fins lucrativos, visando ao interesse comum, e a iniciativa de sua constituição deve partir dos trabalhadores. Os cooperativados são portadores de autonomia e de controle sobre a sua força de trabalho, no entanto, percebe-se que algumas cooperativas são originadas do interesse de empresários e intermediários, estimulados por gestor público ou privado, promovendo a fraude, indo de encontro aos princípios norteadores do sistema cooperativo.

Em Feira de Santana, a contratação da força de trabalho para a Estratégia de Saúde da Família vem ocorrendo por meio de cooperativa de trabalho patrocinada pelo gestor municipal. Dessa forma, a constituição dessa cooperativa não é da iniciativa dos trabalhadores (médicos, enfermeiros e cirurgiões-dentistas), mas sim uma estratégia jurídica adotada pelo gestor municipal para a contratação desses profissionais. Essa estratégia de contratação impõe aos trabalhadores a sua vinculação de forma compulsória à cooperativa de trabalho, tendo como consequência a perda da sua autonomia, a subordinação aos interesses do contratante (gestor municipal) em especial, as precárias condições de infraestrutura da rede assistencial da ESF e a abdicação aos direitos trabalhistas garantidos pela Constituição Federal de 1988 (férias anuais remuneradas, $13^{\circ}$ salário, licença gestação, recolhimento de FGTS e INSS).

O observado em Feira de Santana contradiz os princípios preconizados na ESF, que estimula a estabilidade de vínculo contratual com a estratégia, condição necessária para a continuidade das atividades assistenciais e o envolvimento, a integração e a construção de uma relação de confiança entre os profissionais de saúde e a comunidade. Para nós está claro que o vínculo contratual adotado pela ESF de Feira de Santana (cooperativa de trabalho, exigência 
do gestor municipal para a contratação dos trabalhadores), bem como a precária infraestrutura da rede assistencial impedem a implementação da ESF no município segundo o preconizado e pode servir de desestímulo aos trabalhadores.

A atual conjuntura da sociedade capitalista intensifica o trabalho, exigindo cada vez mais do trabalhador um enorme consumo da capacidade física e psicológica frente à quantidade de fatores de risco a que se expõe. No entanto, a ausência de trabalho também pode levar o trabalhador ao adoecimento, tanto quanto os ambientes laborais inadequados, a forma de organização do trabalho, a falta de valorização do desempenho e a participação insatisfatória nas decisões.

A jornada diária de trabalho de alguns profissionais de saúde parece interminável, segundo Longhi e Craco (2011), considerando a procura de mais de um vínculo empregatício e a pouca flexibilidade quanto à proteção do trabalhador, favorecendo a insegurança nos projetos de vida e perturbando o equilíbrio emocional.

A prevalência de DPM entre os médicos, enfermeiros e cirurgiões-dentistas não foi tão elevada como a dos estudos de: Araújo, Graça e Araújo (2003), que encontraram uma prevalência de $24,6 \%$ entre os cirurgiões-dentistas de Feira de Santana; Nascimento Sobrinho et al. (2006), de 26,0\% entre os médicos de Salvador; Silva et al. (2011), de 23,6\% entre trabalhadores de enfermagem de um hospital de grande porte do Rio de Janeiro; Kirchof et al. (2009), de 18,7\% entre enfermeiros de um hospital do Rio Grande do Sul; Braga, Carvalho e Binder (2010), de 42,6\% entre trabalhadores da rede básica de saúde de Botucatu; e Moroni e Paz (2011), de 18,7\% entre enfermeiros de um hospital do Estado do Rio Grande do Sul.

A prevalência de DPM detectada nesta pesquisa pode ter sido menor do que a encontrada em outros estudos referenciados na literatura nacional entre profissionais de saúde porque a média de idade observada nos trabalhadores estudados foi maior do que a encontrada nos outros estudos, sabendo-se que a prevalência de DPM é maior entre os indivíduos mais jovens (ARAÚJO; GRAÇA; ARAÚJO, 2003; NASCIMENTO SOBRINHO et al., 2006; SILVA et al., 2011; KIRCHOF et al., 2009; MORONI; PAZ, 2011). Devem-se considerar também as peculiaridades das atividades laborais desenvolvidas na ESF que se caracterizam pela demanda organizada, baixa complexidade dos problemas de saúde identificados e pelo reduzido número de situações de urgência e emergência que minimizam a pressão da demanda da clientela sobre os profissionais de saúde (NASCIMENTO SOBRINHO et al., 2006; BRAGA; CARVALHO; BINDER, 2010).

Entretanto, deve-se salientar ainda a necessidade de melhoria das características de trabalho da ESF priorizando mais investimentos na organização dos serviços, na infraestrutura, nos materiais e nos recursos humanos, visando à qualidade dos serviços prestados ao usuário e consequentemente, a satisfação do trabalhador que atua nessa estratégia.

Na triagem para detecção de abuso no consumo de bebidas alcoólicas, foi considerado positivo ao teste CAGE os sujeitos que apresentaram escore maior ou igual a 02 respostas positivas, observando que não houve casos na categoria de enfermeiros, assemelhando-se aos estudos de Sousa (2010), no qual $75,2 \%$ dos profissionais de enfermagem informaram não fazer uso de bebidas alcoólicas. Os médicos pesquisados apresentaram prevalência menor do que a registrada nos estudos de Nascimento Sobrinho et al. (2006), de 5,8 \%, e maior do que a encontrada nos estudos de Gomes (2011), de 1,3\%.

Os resultados obtidos no teste CAGE aplicado aos cirurgiões-dentistas apresentaram a maior prevalência de positivos comparados às categorias estudadas e a outros estudos (NASCIMENTO SOBRINHO et al., 2006; GOMES, 2011).

Entretanto, deve-se analisar com cautela esses resultados, pois esta prevalência pode estar relacionada ao menor número de participantes desta categoria do estudo (33 sujeitos), o que pode superestimar a prevalência, bem como ao conhecimento por parte da população estudada quanto às informações das questões contidas no teste CAGE.

A possibilidade da não resposta pelo conhecimento do questionário utilizado é semelhante entre profissionais da ESF e os demais profissionais da literatura consultada. Um inconveniente dos estudos que utilizam questionários autoaplicáveis é que o sujeito da pesquisa pode não responder a todas as questões colocadas, dificultando o controle das perdas de informação.

O presente artigo ainda apresenta como limitação ter estudado uma população de trabalhadores da Estratégia Saúde da Família restrita a um espaço geográfico, Feira de Santana, Bahia, o que não permite estender seus resultados ao universo dos trabalhadores da ESF no Brasil.

\section{Conclusão}

Os profissionais que atuam na ESF de Feira de Santana são predominantemente do sexo feminino, adultos jovens e casados. Possuem vínculo cooperativado, sugerindo uma falsa ideia de estabilidade contratual e autonomia, carga horária semanal elevada e mais de um vínculo empregatício, configurando-se uma sobrecarga de trabalho que pode influenciar no seu cotidiano profissional e na qualidade de vida. 
A prevalência de DPM e de profissionais positivos ao teste CAGE foi menor do que a encontrada em outros estudos referenciados na literatura nacional. No entanto, com o intuito de proteger e promover a saúde dos trabalhadores, deve-se considerar: a necessidade de alterar a forma de contratação da ESF de Feira de Santana, priorizando a contratação dos trabalhadores por concurso público, o que representa a garantia de estabilidade e vínculo com a ESF e preconiza a continuidade das atividades assistenciais, com interação entre os profissionais de saúde e a comuni- dade; a melhoria da remuneração do trabalhador da ESF com a implantação de Planos de Cargos, Carreira e Salários (PCCS); mais investimentos na organização dos serviços, na infraestrutura, nos materiais e nos recursos humanos, visando à qualidade dos serviços prestados ao usuário e à satisfação do trabalhador que atua na ESF.

Os resultados apresentados estimulam novas investigações para caracterizar mais precisamente as condições de trabalho e saúde dos trabalhadores da Estratégia de Saúde da Família.

\section{Contribuições de autoria}

Todos os autores tiveram contribuição substancial no projeto e no delineamento, no levantamento de dados, na análise e na interpretação, na elaboração do manuscrito, na revisão crítica e na aprovação final da versão a ser publicada.

\section{Referências}

ANTUNES, R. Adeus ao trabalho? Ensaios sobre as metamorfoses e a centralidade do mundo do trabalho. São Paulo: Cortez, 1995.

ARAÚJO, T. M.; GRAÇA, C. C.; ARAÚJO, E. Estresse ocupacional e saúde: contribuições do modelo demanda-controle. Ciências \& Saúde Coletiva, v. 8, n. 4, p. 991-1003, 2003.

BARBOSA, G. A. et al. (Coord.). A saúde dos médicos no Brasil. Brasília, DF: Conselho Federal de Medicina, 2007.

BARROS, D. S. et al. Médicos plantonistas de unidade de terapia intensiva: perfil sócio-demográfico, condições de trabalho e fatores associados à síndrome de burnout. Revista Brasileira de Terapia Intensiva, v. 20, n. 3, p. 235-240, 2008.

BODSTEIN, R. Atenção básica na agenda da saúde. Cências \& Saúde Coletiva, v. 7, n. 3, p. 401-412, 2002.

BRAGA, L. C.; CARVALHO, L. R.; BINDER, M. C. P. Condições de trabalho e transtornos mentais comuns em trabalhadores da rede básica de saúde de Botucatu (SP). Ciência e Saúde Coletiva, Rio de Janeiro, v. 15, supl. 1, p. 1585-1596, 2010.

BRASIL. Ministério da Saúde. Saúde da família: uma estratégia para a reorientação do modelo assistencial. Brasília, DF, 1997.

. Doenças relacionadas ao trabalho: manual de procedimentos para os serviços de saúde. Brasília, DF, 2001a.

. Secretaria de Atenção à Saúde.

Departamento de Atenção Básica. Saúde do trabalhador. Brasília, DF, 2001b. (Série Cadernos de Atenção Básica).
. Indicadores de gestão do trabalho

em saúde: material de apoio para o Programa de Qualificação e Estruturação da Gestão do Trabalho e da Educação no SUS - ProgeSUS. Brasília, DF, 2007.

BRUGUERA, M. et al. La atención de los medicos hacia el cuidado de su propia salud. Resultados de uma encuesta postal. Medicina Clínica (Barcelona), v. 117, n. 13, p. 492-494, 2001.

CHAVES, M. C.; MIRANDA, A. S. Discursos de cirurgiões-dentistas do Programa Saúde da Família: crise e mudança de habitus na saúde pública. Interface Comunicação Saúde Educação, v. 12, n. 24, p. 153-67, jan./mar., 2008.

COUTINHO, E. S. F.; ALMEIDA-FILHO, N.; MARI, J. Fatores de risco para morbidade psiquiátrica menor: resultado de um estudo transversal em três áreas urbanas do Brasil. Revista de Psiquiatria Clínica, v. 26, n. 5, p. 246-256, 1999.

DEJOURS, C. A loucura do trabalho: estudo de psicopatologia do trabalho. São Paulo: Cortez-Oboré, 1987.

FEIRA DE SANTANA (Município). Secretaria Municipal de Saúde. Plano de saúde de Feira de Santana: plano municipal de saúde, 2005/2008. Feira de Santana, Bahia, 2006.

GOLDBERG, D.; HUXLEY, P. Common mental disorders: a bio-social model. 2 ed. London: Tavistock/ Routledge: 1993.

GOMES, D. J. Condições de trabalho e saúde de trabalhadores do Programa de Atenção em Saúde Mental de Feira de Santana - BA. 2011. 97 f. Dissertação (Mestrado em Saúde Coletiva)- 
Universidade Estadual de Feira de Santana, Feira de Santana, 2011.

HARDING, T. W. et al. Mental disorders in primary health care: a study of their frequency and diagnosis in four developing countries. Psychological Medicine, v. 10, n. 2, p. 231-241, 1980.

KIRCHOF, A. L. C. et al. Condições de trabalho e características sócio-demográficas relacionadas à presença de distúrbios psíquicos menores em trabalhadores de enfermagem. Texto \& Contexto Enfermagem, Florianópolis, v. 18, n. 2, p. 215-223, abr./jun. 2009.

KURZ, R. A máquina universal de Harry Potter: o conceito de trabalho imaterial e o neo-utopismo reduzido à tecnologia. Disponível em: < http://obeco. planetaclix.pt/rkurz208.htm>. Acesso em: 10/12/2012.

LONGHI, M. P.; CRACO, P. F. Percepções de trabalhadores de saúde sobre seu processo de trabalho. In: SEMINÁRIO DO TRABALHO: TRABALHO, EDUCAÇÃO E SOCIABILIDADE, 7, 2010, Marília, SP. Anais... Marília: Unesp, 2010. Disponível em: <http:// www.estudosdotrabalho.org/anais-vii-7-seminariotrabalho-ret-2010/Marcelen Palu_Longhi_Priscila Frederico_Craco_Percepes_de_trabalhadores_de saude_sobre\%20_seu_processo_de_trabalho.pdf $>$. Acesso em: 25 out. 2012.

MACHADO, M. H. Os médicos no Brasil: um retrato da realidade. Rio de Janeiro: Fiocruz, 1997.

MARI, J. J.; WILLIANS, P. A validity study of a psychiatric screening questionnaire (SRQ-20) in primary care in the city of São Paulo. The British Journal of Psychiatry, v. 148, n. 1, p. 23-26, 1986. MASUR, J.; MONTEIRO, M. G. Validation of the "CAGE" alcoholism screening test in a brasilian psychiatric inpatient hospital setting. Brazilian Journal of Medical and Biological Research, v. 16, n. 3, p. 215-218, 1983

MORITA, M. C.; HADDAD, A. E.; ARAÚJO, M. E. Perfil atual e tendências do cirurgião-dentista brasileiro. Maringá: Dental Press, 2010.
MORONI, G.; PAZ, A. A. Transtornos mentais menores em trabalhadores da Estratégia Saúde da Família na Serra Gaúcha. In: SEMINÁRIO INTERNACIONAL SOBRE O TRABALHO NA ENFERMAGEM, 3., 2011, Bento Gonçalves, RS. Anais... Bento Gonçalves: Associação Brasileira de Enfermagem, 2011. Disponível em: < http://www.abeneventos.com. br/3siten/siten-trabalhos/files/0103.pdf > . Acesso em: 25 out. 2012 .

NASCIMENTO SOBRINHO, C. L. et al. Cooperativas de trabalho e medicina. Arquivos do Conselho Regional de Medicina do Paraná, v. 21, n. 81, p. 06-12, 2004.

NASCIMENTO SOBRINHO, C. L. et al. Condições de trabalho e saúde mental dos médicos de Salvador, Bahia, Brasil. Cadernos de Saúde Pública, Rio de Janeiro, v. 22, n. 1, p. 131-140, jun. 2006.

NASCIMENTO SOBRINHO, C. L.; NASCIMENTO, M. A.; CARVALHO, F. M. Transformações no trabalho médico. Revista Brasileira de Educação Médica, Rio de Janeiro, v. 29, n. 2, p. 129-135, maio/ago. 2005.

SILVA, J. L. L. et al. Estresse, trabalho e ambiente: prevalência de transtornos mentais comuns entre trabalhadores de enfermagem de um hospital federal no município do Rio de Janeiro. In: SEMANA CIENTÍFICA DA ESCOLA DE ENFERMAGEM AURORA DE AFONSO COSTA, 15., 2008, Niterói, RJ. Anais eletrônicos... Niterói: UFF, 2008. Disponível em: <www.uff.br/anaissegerenf/premio/texto\%20uff. semana.pdf $>$. Acesso em: 26 out. 2011.

SOUSA, M. N. M. Transtornos mentais comuns em profissionais de enfermagem de um hospital especializado em Feira de Santana - BA. 2010. 80 f. Dissertação (Mestrado em Saúde Coletiva)Universidade Estadual de Feira de Santana, Feira de Santana, 2010.

TUCUNDUVA, L. T. C. M. et al. A síndrome da estafa profissional em médicos cancerologistas brasileiros. Revista da Associação Médica Brasileira, v. 52, n. 2, p. 108-112, 2006. 